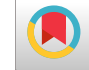

\title{
Potential Adverse Effects of Female and Male Obesity on Fertility: A Narrative Review
}

\author{
Mina Amiri ${ }^{1}$ and Fahimeh Ramezani Tehrani ${ }^{1,{ }^{*}}$ \\ ${ }^{1}$ Reproductive Endocrinology Research Center, Research Institute for Endocrine Sciences, Shahid Beheshti University of Medical Sciences, Tehran, Iran \\ "Corresponding author: Reproductive Endocrinology Research Center, Research Institute for Endocrine Sciences, Shahid Beheshti University of Medical Sciences, Tehran, Iran. \\ Email: fah.tehrani@gmail.com
}

Received 2020 February 12; Revised 2020 September 15; Accepted 2020 September 19.

\begin{abstract}
Context: Despite several studies documenting that obesity affects female and male fertility and leads to multiple adverse reproductive outcomes, the mechanisms involved are not elucidated. We aimed to summarize the potential adverse effects of female and male obesity, as well as the impact of weight loss on their fertility status.

Evidence Acquisition: This review summarizes papers investigating the potential adverse effects of female and male obesity and the impact of weight-loss interventions on fertility among reproductive age populations. PubMed, Scopus, Web of Science, and Google Scholar databases were searched for retrieving studies published up to November 2019 on obesity/overweight among reproductive age populations.

Results: The review of 68 studies revealed that female and male obesity/overweight increases the risk of sub-fecundity and infertility. The destructive effects of female obesity on reproduction are attributed to a variety of ovarian and extra-ovarian factors. In women with overweight or obesity, the time taken to conceive is longer, and they have a decreased fertility rate, increased requirement for gonadotropins, and higher miscarriage rate when compared to those with normal weight. Male obesity may lead to subfertility, mainly because of the disruption of the hypothalamus-pituitary-gonadal (HPG) axis, increased testicular temperature, impairment of the physical and molecular structure of sperm, decreased sperm quality, and erectile dysfunction due to peripheral vascular disease. Most studies recommend lifestyle interventions as first-line therapy in the general population of women and men with obesity and infertility.

Conclusions: This study shows the negative effects of female and male overweight and obesity on fertility. Therefore, educational interventions on the adverse effects of obesity and the benefits of weight reduction, such as increasing pregnancy rate, should be considered for couples seeking fertility.
\end{abstract}

Keywords: Obesity, Overweight, Fertility, Infertility, Pregnancy

\section{Context}

Obesity, a common metabolic disturbance, is defined as an abnormal or excessive accumulation of body fat (1). According to the current guidelines from the US Centers for Disease Control and Prevention (CDC) and the World Health Organization (WHO), a Body Mass Index (BMI) of $\geq$ $25 \mathrm{~kg} / \mathrm{m}^{2}$ is classified as overweight, a BMI of $\geq 30 \mathrm{~kg} / \mathrm{m}^{2}$ as obese, and a BMI of $\geq 40 \mathrm{~kg} / \mathrm{m}^{2}$ as severely obese (2). This metabolic condition that is a rapidly growing threat to the health of populations worldwide plays a major role in the pathophysiology of various diseases, in particular, cardiovascular disorders $(1,3,4)$.

In addition to the cardio-metabolic consequences of obesity, there is evidence demonstrating that female and male obesity/overweight can increase the risk of sub- fecundity and infertility (3-5). Earlier studies have shown that over $40 \%$ of women with menstrual disorders, infertility, and recurrent miscarriages are either overweight or obese (6). Further studies confirm that the prevalence of anovulatory cycles, oligo-amenorrhea, hirsutism, infertility, and/or sexual dysfunction is significantly higher in obese than in normal-weight women $(7,8)$. Several studies conducted on male populations also suggest that male obesity may be associated with reduced fertility and fecundity rates (9). Despite the documentation of a threefold increase in the incidence of obesity in patients with male factor infertility, the impact of male obesity or overweight on fertility has been overlooked (10).

Mechanisms involved in the relationship between excess body fat and reproductive disturbances are complex and multifactorial (3). Female obesity can influence re- 
production through an impairment in the hypothalamuspituitary-gonadal (HPG) axis (hyperandrogenism) and the follicular environment $(3,11,12)$. It is well documented that in women with obesity, the time taken to conceive is longer, and they have a decreased fertility rate, increased requirement for gonadotropins, and elevated miscarriage rate when compared to those with normal weight (4). Also, male obesity may lead to subfertility, mainly because of the disruption of the HPG axis (hypogonadism), increased testicular temperature, impairment of the physical and molecular structure of sperm during both spermatogenesis in the testis and sperm maturation in the epididymis decreased sperm quality, and erectile dysfunction due to peripheral vascular disease $(3,13)$.

While previous studies have shown that lifestyle interventions such as weight loss can improve fertility in women and men with obesity, mainly increasing the chance of spontaneous pregnancy, controversial results have been documented regarding the effect of these interventions on reproduction $(14,15)$. On the other hand, there is evidence demonstrating that aggressive low-calorie diets and surgical techniques for weight reduction may have negative effects on fertility $(8,15)$.

Despite numerous studies on the relationship between obesity and reproduction, data available are conflicting and inconclusive. Furthermore, the exact mechanisms involved in this association and the impact of weight loss on reproduction have not been elucidated (16-20). This review summarizes the potential adverse effects of female and male obesity, as well as the impact of weight loss on fertility status.

\section{Evidence Acquisition}

This study reviewed papers investigating the potential adverse effects of female and male obesity and the impact of weight loss on fertility among reproductive age populations. PubMed, Scopus, Web of Science, and Google Scholar databases were searched for retrieving studies published up to November 2019 on obesity/overweight among reproductive age populations. All databases were searched using the combination of the following keywords: "obesity" OR "obese" OR "overweight" OR "body mass index" OR "BMI" OR "body fat" OR "body weight") AND ("fertility" OR "fecundity" OR "fecundability" OR "infertility" OR "infertile" OR "reproduction" OR "reproductive" OR "conceive" OR "pregnancy" OR "pregnant"). The final selection of papers was made based on their relevance with the effects of female and male obesity and weight loss on fertility in this age group.

\section{Results}

Of 240,000 records retrieved by searching the databases, a total of 68 relevant studies were selected for this review. We first summarize the results of studies investigating the associations between male/female obesity and reproduction. Next, we discuss the effects of female/male obesity on assisted reproductive technology. Finally, the influences of weight reduction on female/male fertility are discussed.

\subsection{Female Obesity and Reproduction}

Obesity/overweight is common among reproductiveaged women. The destructive effects of obesity on reproductive function are well documented. Many obese women experience menstrual irregularity and infertility due to ovulatory disorders and endometrial pathology (4). Obesity is considered a well-known risk factor for infertility in women, which can affect both offspring's health and maternal health $(12,21)$.

One study estimated that the risk of infertility is $78 \%$ and $27 \%$ in reproductive-aged women with obesity and overweight, respectively, compared to those with normal BMI (18.5 - 25) (21). Another prospective cohort study of approximately 3000 women showed that with each unit $\left(\mathrm{kg} / \mathrm{m}^{2}\right)$ increment in BMI, the odds of spontaneous pregnancy declined by $10 \%$ (22). Some studies showed an association between obesity and decreased pregnancy and livebirth rates in infertile women seeking fertility treatments $(23,24)$, indicating that weight loss in these obese women can be considered an appropriate strategy before infertility treatment (3).

\subsubsection{Pathophysiology}

Female obesity may influence fertility through complex and multiple mechanisms, which have not yet been completely understood (Box 1) (25). The negative effects of female obesity on reproduction are attributed to ovarian follicular recruitment, oocyte development and quality, oocyte fertilization, and embryo development and implantation (12). Fertility is influenced by a variety of ovarian and extra-ovarian factors. Obesity impacts neuroendocrine and ovarian functions, leading to reduced ovulatory cycles and fertility rates (26). It can also influence ovarian reserve, a surrogate marker of female fertility (27). Studies on polycystic ovary syndrome (PCOS) support the association of obesity with hyperandrogenism and anovulation among reproductive-aged women. Evidence demonstrating the role of insulin resistance in the association between PCOS and obesity shows that elevated insulin levels can stimulate androgen production by the ovaries. In addition, 
higher triglyceride and fatty acid levels, which are associated with obesity and insulin resistance, can induce androgen production in predisposed women (14). Obese women have several abnormalities in their sex steroid concentrations. They also have lower sex hormone-binding globulin (SHBG) levels and higher androgen and estrogen levels than those with normal body weight $(8,28)$. Obese women often have higher circulating levels of insulin and androgen; androgen is aromatized to estrogens at higher rates in the periphery due to the excess of adipose. Estrogens can result in negative feedback on the HPG axis and gonadotropin impairment (increased secretion of luteinizing hormone), which leads to ovulatory dysfunction and menstrual abnormalities (25).

Box 1. The Main Mechanisms Involved in the Effects of Female Obesity/Overweight on Fertility

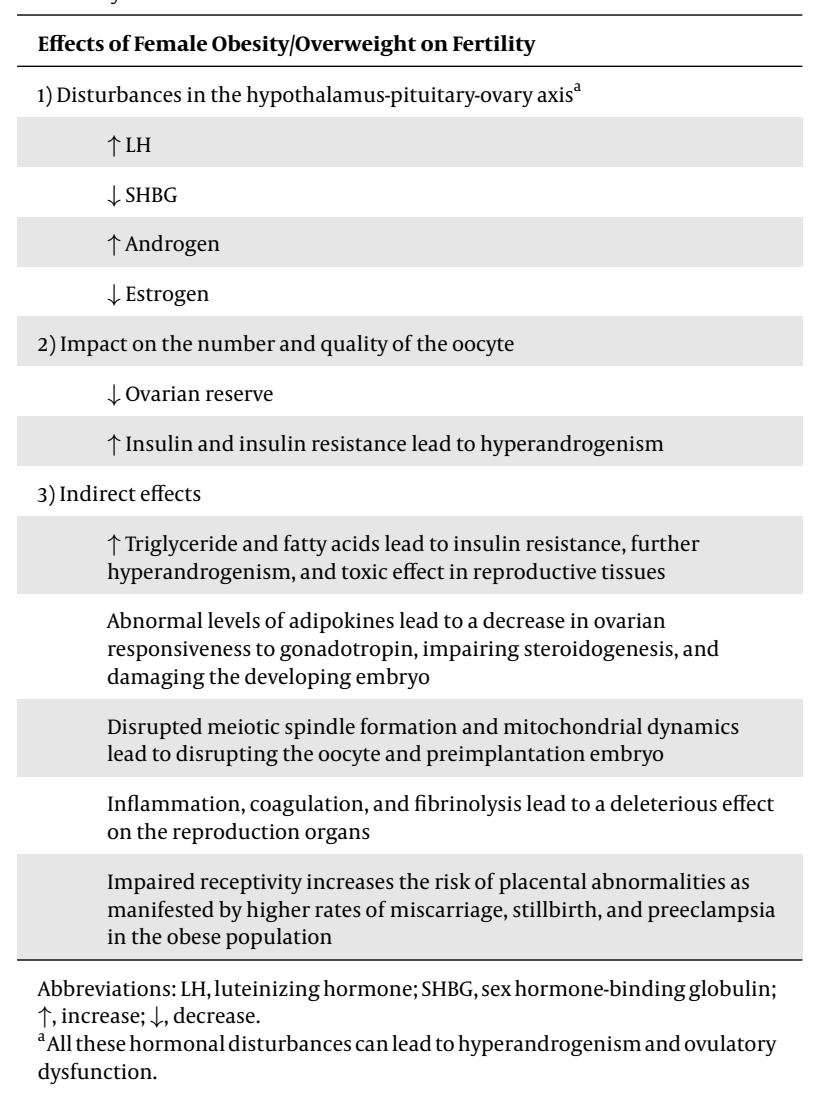

Obesity can also alter the profiles of specific hormones such as insulin, adipokines, growth hormone (GH), and insulin-like growth factor binding proteins (IGFBP), leading to impairments in a woman's fertility. There are associations between energy metabolism and fertility in females (4). Recent studies suggest that the normal functioning of adipose tissue and its hormones (adipokines) is critical to preserve the integrity of the HPG axis. In- deed, the abnormal levels of adipokines, viz. leptin, can be associated with insulin resistance (IR). Subsequently, hyperinsulinemia and insulin resistance can affect ovarian and endometrium function by inducing hyperandrogenism and alterations in steroidogenesis (4). Obesity can influence the oocytes and preimplantation embryo by disrupting meiotic spindle formation and mitochondrial dynamics. In addition, an increase in free fatty acids may lead to a toxic effect in reproductive tissues, causing cellular and chronic inflammatory states (5). Increased adiposity is also related to inflammation, coagulation, and fibrinolysis, and these changes may have a deleterious effect on reproduction (27). Furthermore, obesity can correlate with impaired receptivity, thereby increasing the risk of placental abnormalities as manifested by higher rates of miscarriage, stillbirth, and preeclampsia in obese populations (5).

\subsubsection{Impact of Female Obesity on Abnormal Uterine Bleeding}

Obesity is considered an important risk factor for menstrual cycle disorders (8). Data show the earlier onset of menarche in obese women than in normal-weight counterparts (26). Subsequently, the onset of ovarian failure and menopause is earlier in obese than in normal-weight women (29). A higher incidence of longer and more irregular menstrual cycles has been reported in obese women aged 43 - 55 years (30). The rates of menstrual cycle disturbances and oligo-anovulation are three-fold higher in obese women than in those with normal weight; however, a study has shown the negative effects of obesity on women's reproductive health, even in women without ovulatory or menstrual dysfunction (31).

Although the precise mechanism of the effect of obesity on menstrual irregularity has not yet been elucidated, endocrine disorders, viz. elevated levels of estrogen, via the peripheral conversion of androgens to estrogen play a substantial role. Moreover, the role of adipokines, i.e., leptin, in the regulation of gonadotropin secretion and the induction of the development of pubertal stages, is well documented (32). Furthermore, hyperinsulinemia and insulin resistance in obese women can intensify the activity of the luteinizing hormone (LH) in granulosa cells, leading to premature luteinization and consequently, follicular arrest, impaired endometrium, and eventually menstrual cycle disturbances $(4,27)$.

\subsubsection{Impact of Female Obesity on Oocytes}

Data available support the impact of overweight/obesity on oocyte quality (5). Diet-induced obesity (DIO) in mouse models demonstrated that the ovaries that have more apoptotic follicles and oocytes are smaller and 
less likely to be mature (33); these abnormal oocytes often have high rates of meiotic aneuploidy with fragmented, disorganized meiotic spindles and improperly aligned chromosomes on the metaphase plate (34). Indeed, obesity can alter the mitochondrial function of the oocyte. In addition, evidence shows endoplasmic reticulum (ER) stress in the obese state. Moreover, the cumulus-oocyte complexes of mice fed with a high-fat diet show that the increased expression of ER stress markers can increase granulosa cell apoptosis. It also seems that obesity influences the oocyte and pre-implantation embryo by disrupting meiotic spindle formation and mitochondrial dynamics (5). Obesity can also be associated with oocyte damage through a lipotoxicity effect on the oocyte. Inflammatory pathways are essential in reproductive events such as follicle rupture at the time of ovulation and the invasion of trophoblasts to the receptive endometrium, and the altered inflammatory milieu in obese women can most likely exert an influence on these processes (5). In addition, the abnormal levels of leptin in obese women may impair follicle development, ovulation, and oocyte maturation (35).

A retrospective analysis conducted on infertile couples revealed that overweight women $\left(\mathrm{BMI}>25 \mathrm{~kg} / \mathrm{m}^{2}\right)$ had relatively fewer metaphase II oocytes collected (36). Similarly, a prospective study could reduce the number of oocytes, the concentration of intra-follicular human chorionic gonadotrophin (hCG), and the number of mature (metaphase II) oocytes retrieved in obese women (BMI > $30 \mathrm{~kg} / \mathrm{m}^{2}$ ), although these reductions were not statistically significant (37).

The fertilization rate is a surrogate marker of oocyte quality. Some studies showed a decreased rate of fertilization in overweight or obese women, compared to normalweight ones (38-40), whereas others reported no such effect $(41,42)$. However, investigators suggest that increased rates of pregnancy loss and miscarriage can be attributed to impaired oocyte quality (43).

\subsubsection{Impact of Female Obesity on Endometrium and Implanta-} tion

The endometrium is another potential target of the abnormal environment created by obesity (12). Several studies evaluating the impact of obesity on endometrial development, uterine receptivity, implantation, and miscarriage, have documented inconsistent results; some showed a reduction in the implantation rate among obese women $(20,44,45)$, whereas others reported no such effect $(41,46)$.

A recent study showed that compared to normalweight women, obese women receiving oocytes from normal-weight donors were less likely to conceive. This study suggested that the endometrium of these women exhibits dysregulated genes related to molecular functions, such as protein binding, and biologic processes, including development, morphogenesis, and immunity (47). Indeed, decidualization defects in obese women may contribute to compromised endometrial receptivity and poor implantation. In addition, in vitro studies have demonstrated that leptin can affect steroidogenic pathways in granulosa cells leading to downstream effects on endometrial receptivity and embryo implantation (5). Robker (48) suggested that a reduction in oocyte developmental competence may impair the developmental potential of the embryo, which may lead to an impaired implantation rate and subsequent abnormal implantation/trophoblastic invasion.

\subsubsection{Impact of Female Obesity on Egg}

It is documented that both oocyte maturation and metabolism are impaired due to obesity, which, in turn, negatively affect further development (31). In fact, a fine hormonal balance regulates follicular development and oocyte maturation, and obesity can alter the hormonal environment (49). In addition, obesity can induce elevations in insulin, glucose, free fatty acids, and $\mathrm{C}$ reactive protein (CRP), and changes in adipokines apparently impact the developmental competence of the oocyte $(31,35)$.

Animal studies have demonstrated that abnormal concentrations of leptin can impair follicle development, ovulation, and oocyte maturation (35). In addition, the changed levels of leptin in obese females can also affect steroidogenesis, directly damaging the developing embryo (5). In vivo studies showed that any of these hormones or metabolites can negatively influence oocyte developmental competence (31). In agreement with previous studies, PCOS studies demonstrated that obese PCOS women with hyperinsulinemia and impaired glucose tolerance had lower fertilization and implantation rates than their non-obese PCOS counterparts (50). Nevertheless, further research is required for a deeper insight into the underlying mechanisms of the effects of obesity on oocyte development (31).

\subsubsection{Obesity and Time to Pregnancy}

Obesity is associated with increased time taken to conceive (47). A study from the USA showed reduced fecundity in both overweight (OR $0.92,95 \%$ confidence interval [CI]: 0.84 - 1.01) and obese (OR 0.82, 95\% CI: 0.72 - 0.95) women compared to women with normal weight (51). A study conducted by van der Steeg et al. (22) on 3,029 couples demonstrated a linear decline in the probability of spontaneous pregnancy in women with a BMI of 29 ; they 
also found that each unit increase in BMI was associated with a $4 \%-5 \%$ lower pregnancy rate. A prospective study showed that the risk of infertility was threefold higher in obese women than in non-obese ones and concluded that obese women needed a longer time to conceive than their normal-weight counterparts (52). There are data demonstrating that time taken to conceive spontaneous pregnancy increases in obese women, even those with regular ovulation (12).

\subsection{Male Obesity and Reproduction}

Obesity has multifactorial effects on male reproductive function (53). Male factors are estimated to contribute to up 50\% of subfertility cases, with $31.5 \%$ being attributed solely to a male factor (10). Obesity in men is most likely associated with reduced fertility and fecundity rates (9). Population-based studies have reported the increased chance of abnormal semen parameters and subfertility among overweight and obese men $(18,54,55)$.

A recent study showed a higher prevalence of low ejaculatory volume and oligo-zoospermia in obese men with increased BMI and waist circumference (55). A crosssectional study revealed that increasing body adiposity in men was associated with lower sperm concentration, a higher percentage of abnormal sperm morphology, and increased body adiposity (56). Changes in sperm function as a result of obesity may be associated with impaired embryo development and reduced live birth rates (57).

Most available studies have been conducted on men undergoing assisted reproductive technology (ART) treatments. A meta-analysis of 11 studies showed that high BMI in men was associated with a significant reduction in the pregnancy rate and live birth rate per in vitro fertilizationintracytoplasmic sperm injection (IVF-ICSI) treatment cycle; they concluded that male BMI could be an important factor influencing the IVF-ICSI outcome (58). Despite the extensive knowledge on the association between obesity and female fertility, the impact of male obesity or overweight on fertility remains unclarified.

\subsubsection{Pathophysiology}

The main mechanisms responsible for the effects of male obesity on their infertility mostly remain ambiguous and unexplained. However, it is suggested that obesity may negatively influence reproductive function among men through numerous mechanisms, including lower serum testosterone, higher serum estradiol levels, impaired spermatogenesis and erectile dysfunction (due to atherogenic effect on peripheral vasculature), increased testicular heat, and elevated inflammatory mediators (Box 2) $(59,60)$.

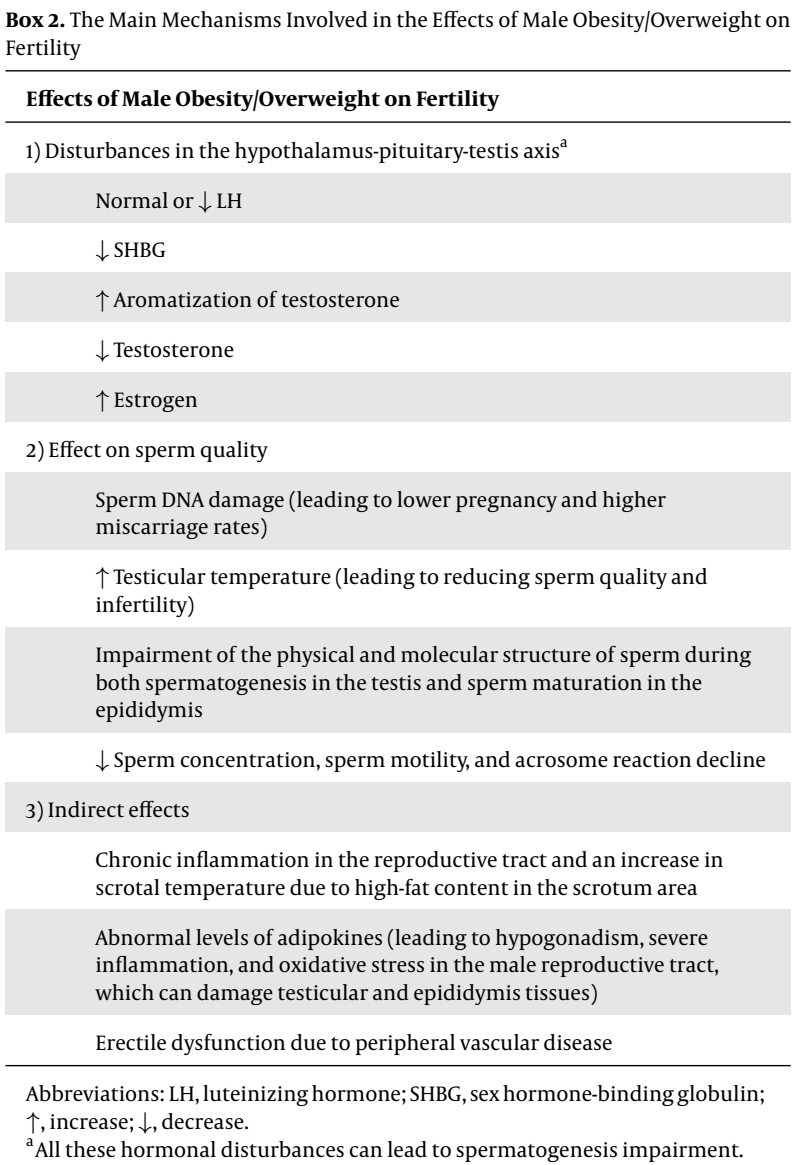

$\downarrow$ Sperm concentration, sperm motility, and acrosome reaction decline

Chronic inflammation in the reproductive tract and an increase in scrotal temperature due to high-fat content in the scrotum area

Abnormal levels of adipokines (leading to hypogonadism, severe inflammation, and oxidative stress in the male reproductive tract, which can damage testicular and epididymis tissues)

Erectile dysfunction due to peripheral vascular disease

Abbreviations: LH, luteinizing hormone; SHBG, sex hormone-binding globulin; $\uparrow$, increase; $\downarrow$, decrease.

${ }^{a}$ All these hormonal disturbances can lead to spermatogenesis impairment.

In normal men, gonadotropin-releasing hormone (GnRH) is produced and released from the hypothalamus, leading to the stimulation and production of the folliclestimulating hormone (FSH) and $\mathrm{LH}$ from their anterior pituitary. FSH and LH then act on the testicle to stimulate spermatogenesis and steroidogenesis, respectively. Obese men have normal or decreased LH levels, decreased total testosterone levels, and reduced SHBG levels (60).

Male obesity can also influence fertility through increased testicular temperature with sedentary lifestyles, increasing estrogen production in fat with the disruption of the HPG axis (3). Adipokines produced by adipose tissue may induce severe inflammation and oxidative stress in the male reproductive tract, which can damage testicular and epididymis tissues (60). Previous studies showed that leptin has a direct effect on the regulation of testosterone production in the testicle, demonstrating a correlation between high circulating leptin levels and hypogonadism (60). However, it should be kept in mind that the effects of male obesity on fertility are likely multifactorial. In addition, obesity is often associated with metabolic dis- 
orders, including metabolic syndrome, hyperlipidemia, cardiovascular disease, and proinflammatory state, all of which can predispose to male subfertility (57).

\subsubsection{Impact of Male Obesity on Sperm Quality}

There is strong evidence that obesity can impair the physical and molecular structure of sperm during both spermatogenesis in the testis and maturation in the epididymis. Compared to normal-weight men, those with overweight or obesity may have decreased sperm quality (in concentration and motility, declined acrosome reaction, and increased sperm DNA damage) and lower embryo implantation rates. Indeed, the excessive visceral adiposity in obese males can result in changes in their hormone levels, resulting in chronic inflammation in the reproductive tract and increased scrotal temperature due to high-fat content in the scrotum area, all of which can reduce sperm quality and cause male infertility risk (60).

Human and animal models have demonstrated the association of male obesity with sperm parameters, although the impact of male obesity on sperm count, motility, and morphology in humans is controversial (57). A meta-analysis of 25 studies showed that in men with abnormal weight, all sperm parameters, including total sperm count, sperm concentration, and semen volume, decreased by $2.4 \%, 1.3 \%$, and $2.0 \%$, respectively, compared to normal-weight men for every five-unit increase in BMI; these findings suggest that obesity may be a detrimental factor in male infertility (61).

\subsubsection{Male Obesity and Infertility}

Simultaneously with the epidemic of obesity, male infertility is increasing rapidly worldwide, suggesting obesity may be a detrimental factor in male infertility (61), which can increase male infertility risk, mainly through sex hormone imbalances (hypogonadism), detrimental effects on spermatogenesis, and abnormal production of inflammatory factors (60). A recent study revealed that obesity was associated with approximately $20 \%$ increased cases of subfertility and infertility (61). A large cohort of 26,303 planned pregnancies showed that the OR of infertility at 12 months was 1.2 (95\% CI:1.04 - 1.38) in overweight men and 1.36 (95\% CI: 1.13 - 1.63) in obese men, compared to their normal-weight counterparts (62). Another study from the USA reported an association between increased BMI and infertility and showed that each $3 \mathrm{~kg} / \mathrm{m}^{2}$ increase in BMI was associated with a 1.2-fold increase in the risk of subfertility (63). Although the impact of obesity on female fertility is well understood, male infertility as a result of obesity has been overlooked, despite a threefold increase in the incidence of obesity in patients with male factor infertility (64).

\subsection{Female/Male Obesity and Assisted Reproductive Technology}

\subsubsection{ART in Infertile Obese Women and Men}

Several studies suggest that both male and female obesity may have adverse effects on the pregnancy of ART, such as the poor quality of the oocyte and sperm, decreased live birth rate, cycle cancellation, and miscarriage (19, 20, 53, $65,66)$.

It is well documented that sex steroid hormone dysregulation may lead to longer periods of follicular stimulation, more follicular asynchrony, cycle cancellation, and lower live birth rates among obese women who undergo IVF as their ART compared to normal weight infertile patients. Hence, each one-unit increase in BMI decreases the probability of pregnancy in IVF by $2.2 \%-4.3 \%$. In addition, the implantation rates of obese women are lower than those with normal weight. The impact of obesity may also contribute to aberrant gene expression regulation during the window of implantation (11). Moreover, these women often have a higher risk of miscarriage after an ART conception and are less likely to achieve a live birth after IVF, compared to normal-weight women; these risks may be a result of abnormal endometrial development and implantation (12), indicating that overweight and obesity can be associated with destructive outcomes for women undergoing IVF due to the poor oocyte quality, lower pre-implantation rate, and uterine receptivity (20). A recent meta-analysis of 49 studies showed that overweight and obese women have a significantly lower live birth rate following ART than have women with normal BMI (19).

Male obesity/overweight can also have adverse outcomes for men undergoing IVF, mainly due to abnormal sperm parameters (55). A meta-analysis of 30 studies (a total of 115,158 male participants) showed that obese men had a higher odds ratio of infertility, whereas the odds of live births per cycle of ART reduced in these men; they also had a $10 \%$ increase in the absolute risk of non-viable pregnancy. This meta-analysis demonstrated that obese men had an increased percentage of sperm with low Matrix Metalloproteinase (MMP), DNA fragmentation, and abnormal morphology. These findings suggest that male obesity is associated with reduced reproductive potential (54). A further meta-analysis of 10 cohort studies, including 5,262 male participants, showed that overweight or obesity had no significant impact on the clinical pregnancy rate, including IVF, ICSI, live birth, and sperm concentration compared to those with normal weight following IVF/ICSI treatments (18). Despite the data available regarding the impact of male BMI and ART outcomes, much controversy exists in 
the results of these studies, and the role of male obesity in the process of ART is not elucidated, indicating the need for further investigations.

\subsubsection{ART Outcomes in Obese Donor Oocyte/Sperm Recipients}

Female obesity can impair the reproductive outcome of ovum donation, probably due to reduced uterine receptivity (67). Some researchers suggest that alterations in endometrial gene expression in the peri-implantation period differ in obese women, compared to their normal-weight counterparts $(12,68)$. Several studies have evaluated the reproductive outcome of recipients of a donated oocyte, with conflicting results $(45,67,69,70)$. While two studies demonstrated BMI-related impact on the measures of reproductive success (embryonic implantation rate, clinical pregnancy rate, miscarriage rate, and chances of live birth) $(69,70)$, others did not $(45,70)$. Finally, a recent meta-analysis of five studies demonstrated that the chance of pregnancy after IVF did not differ between obese donor oocyte recipients and those in the normal BMI range, the findings suggesting that obesity does not affect IVF outcomes in women using donor oocytes. Hence, it seems that oocyte quality rather than endometrial receptivity is the important factor influencing IVF outcomes in obese women using autologous oocytes (68).

Two studies evaluated the effects of male BMI on fertility. One study demonstrated that the BMI of men does not correlate with sperm parameters although it influences the quality of the embryos so that it impacts the pregnancy rate (71). Similarly, another study showed that increased male BMI does not affect the live birth success (72); however, these two studies have not assessed the effects of male BMI on reproductive outcomes in obese donor sperm recipients.

\subsection{Weight Reduction and Female/Male Fertility}

Therapeutic interventions for weight reduction, including conservative (lifestyle modifications and pharmacologic agents) or surgical approaches can be associated with an increase in female and male fertility, mainly through improving hormonal profiles, the spontaneous return of ovulation, and increasing oocyte and semen quality $(8,15,28,49)$.

Despite the controversy about the effects of weight reduction, particularly with surgical methods $(8,28)$, on improving reproduction, most available studies support the positive association between weight reduction and improved chances of pregnancy and live birth among obese infertile women $(3,21,28)$. The Danish National Birth Cohort among 2,374 women showed that those who were overweight or obese and had lost or maintained a weight between pregnancies had, on average, 5.50 days' shorter time to pregnancy for each $1 \mathrm{~kg}$ decrement in weight (21). A recent meta-analysis of 40 studies showed that nonsurgical weight reduction strategies can improve reproductive outcomes in both infertile men and women (3).

Despite the existing studies assessing the benefits of lifestyle interventions as first-line therapy in the general population of women with obesity and infertility, these interventions are generally the preferred approaches to preconception weight loss, compared to bariatric surgery or pharmacologic agents (14). Weight reduction can improve reproductive outcomes through regulating menstrual cycles and increasing the chance of spontaneous ovulation and conception in anovulatory overweight and obese women (35).

Generally, pharmacologic agents such as metformin are not prescribed to improve fertility in infertile women mainly because of the potential consequences on the health of mothers and their offspring (14). In addition, bariatric surgery cannot be recommended as a first-line fertility treatment for obese women. According to the American Society for Metabolic and Bariatric Surgery recommendations, bariatric surgery should be considered for a person with $\mathrm{BMI} \geq 40 \mathrm{~kg} / \mathrm{m}^{2},>100$ pounds overweight, or BMI $\geq 35 \mathrm{~kg} / \mathrm{m}^{2}$ and at least two obesity-related comorbidities (13). The exact mechanism of increased fertility after weight reduction with bariatric surgery is yet unknown. On the other hand, there is evidence indicating the negative effects of bariatric surgery on fertility (8, 73). The most commonly performed bariatric procedure is Roux-Y gastric bypass; restrictive and malabsorptive operations are not common since these techniques are often associated with a high risk of long-term adverse effects (8). There is also evidence demonstrating that a balance in dietary fat, moderation of physical activity, and management of a healthy body habitus can improve the semen quality and birth outcomes; however, further detailed studies are needed to clarify the impact of these interventions in subfertile males (15).

Several guidelines recommend lifestyle changes before infertility treatments, such as IVF (74). However, the optimal weight-loss threshold that should be recommended to improve fertility and pregnancy outcomes has not yet been determined. Moreover, it is unknown whether specific lifestyle modifications have a direct effect on fertility in addition to promoting weight loss (14). Further welldesigned and powerful randomized controlled trials may clarify these uncertainties.

The main strengths of this study were the relatively large number of studies reviewed, and the assessment of the diverse effects of obesity on fertility in both male and 
female populations. However, this review had some limitations that should be considered. First, a limited number of studies have assessed the effects of obesity on male infertility, with controversial results. Second, most studies reviewed for the study were conducted on individuals undergoing ART, and there was a lack of studies to explain the association between obesity and fertility in the general population. Finally, there was insufficient data on reproduction outcomes in obese donor oocyte/sperm recipients. Therefore, more well-designed studies are needed to address the effects of male and female obesity on reproduction in different populations, in particular obese donor oocyte/sperm recipients.

\section{Conclusions}

This review showed the negative effects of female and male overweight and obesity on their fertility, indicating the need for educational interventions based on the adverse effects of obesity and the benefits of weight reduction in increasing pregnancy rates among couples suffering from infertility.

\section{Footnotes}

Authors' Contribution: Mina Amiri conceptualized and designed the study, participated in the literature search, and drafted the manuscript. Fahimeh Ramezani Tehrani conceptualized the study and supervised the manuscript. All authors approved the final version of the manuscript.

Conflict of Interests: The authors had no conflict of interest to declare.

Ethical Approval: The ethical approval code was IR.SBMU.Endocrine.REC.1398.007.

\section{Funding/Support: None.}

\section{References}

1. Jayawardena R, Ranasinghe P, Ranathunga T, Mathangasinghe $Y$, Wasalathanththri S, Hills AP. Novel anthropometric parameters to define obesity and obesity-related disease in adults: a systematic review. Nutr Rev. 2020;78(6):498-513. doi: 10.1093/nutrit/nuz078. [PubMed: 31841153].

2. Chooi YC, Ding C, Magkos F. The epidemiology of obesity. Metabolism. 2019;92:6-10. doi:10.1016/j.metabol.2018.09.005. [PubMed:30253139].

3. Best D, Avenell A, Bhattacharya S. How effective are weight-loss interventions for improving fertility in women and men who are overweight or obese? A systematic review and meta-analysis of the evidence. Hum Reprod Update. 2017;23(6):681-705. doi: 10.1093/humupd/dmx027. [PubMed: 28961722].

4. Silvestris E, de Pergola G, Rosania R, Loverro G. Obesity as disruptor of the female fertility. Reprod Biol Endocrinol. 2018;16(1):22. doi: 10.1186/s12958-018-0336-z. [PubMed: 29523133]. [PubMed Central: PMC5845358].
5. Broughton DE, Moley KH. Obesity and female infertility: potential mediators of obesity's impact. Fertil Steril. 2017;107(4):840-7. doi: 10.1016/j.fertnstert.2017.01.017. [PubMed: 28292619].

6. Rogers J, Mitchell GJ. The relation of obesity to menstrual disturbances. $N$ Engl $J$ Med. 1952;247(2):53-5. doi: 10.1056/NEJM195207102470204. [PubMed: 14941282].

7. Hartz AJ, Barboriak PN, Wong A, Katayama KP, Rimm AA. The association of obesity with infertility and related menstural abnormalities in women. Int J Obes. 1979;3(1):57-73. [PubMed: 528119].

8. Slopien R, Horst N, Jaremek JD, Chinniah D, Spaczynski R. The impact of surgical treatment of obesity on the female fertility. Gynecol Endocrinol. 2019;35(2):100-2. doi: 10.1080/09513590.2018.1500536. [PubMed: 30599791].

9. Sundaram R, Mumford SL, Buck Louis GM. Couples' body composition and time-to-pregnancy. Hum Reprod. 2017;32(3):662-8. doi: 10.1093/humrep/dex001. [PubMed: 28158570]. [PubMed Central: PMC5400044]

10. Agarwal A, Mulgund A, Hamada A, Chyatte MR. A unique view on male infertility around the globe. Reprod Biol Endocrinol. 2015;13:37. doi: 10.1186/s12958-015-0032-1. [PubMed: 25928197]. [PubMed Central: PMC4424520].

11. Pantasri T, Norman RJ. The effects of being overweight and obese on female reproduction: a review. Gynecol Endocrinol. 2014;30(2):90-4. doi: 10.3109/09513590.2013.850660. [PubMed: 24188449].

12. Jungheim ES, Travieso JL, Hopeman MM. Weighing the impact of obesity on female reproductive function and fertility. Nutr Rev. 2013;71 Suppl 1:S3-8. doi: 10.1111/nure.12056. [PubMed: 24147921]. [PubMed Central: PMC3813308].

13. Craig JR, Jenkins TG, Carrell DT, Hotaling JM. Obesity, male infertility, and the sperm epigenome. Fertil Steril. 2017;107(4):848-59. doi: 10.1016/j.fertnstert.2017.02.115. [PubMed: 28366411].

14. Belan M, Harnois-Leblanc S, Laferrere B, Baillargeon JP. Optimizing reproductive health in women with obesity and infertility. CMAJ. 2018;190(24):E742-5. doi: 10.1503/cmaj.171233. [PubMed: 29914911]. [PubMed Central: PMC6008192].

15. Hayden RP, Flannigan R, Schlegel PN. The Role of Lifestyle in Male Infertility: Diet, Physical Activity, and Body Habitus. Curr Urol Rep. 2018;19(7):56. doi: 10.1007/s11934-018-0805-0. [PubMed: 29774489].

16. Thomsen L, Humaidan P, Bungum L, Bungum M. The impact of male overweight on semen quality and outcome of assisted reproduction. Asian J Androl. 2014;16(5):749-54. doi: 10.4103/1008-682X.125398. [PubMed: 24759576]. [PubMed Central: PMC4215681].

17. Wogatzky J, Wirleitner B, Stecher A, Vanderzwalmen P, Neyer A, Spitzer D, et al. The combination matters-distinct impact of lifestyle factors on sperm quality: a study on semen analysis of 1683 patients according to MSOME criteria. Reprod Biol Endocrinol. 2012;10:115. doi: 10.1186/1477-7827-10-115. [PubMed: 23265183]. [PubMed Central: PMC3575231]

18. Le W, Su SH, Shi LH, Zhang JF, Wu DL. Effect of male body mass index on clinical outcomes following assisted reproductive technology: a meta-analysis. Andrologia. 2016;48(4):406-24. doi: 10.1111/and.12461. [PubMed: 26276351].

19. Supramaniam PR, Mittal M, McVeigh E, Lim LN. The correlation between raised body mass index and assisted reproductive treatment outcomes: a systematic review and meta-analysis of the evidence. Reprod Health. 2018;15(1):34. doi: 10.1186/s12978-018-0481-z. [PubMed: 29486787]. [PubMed Central: PMC5830337].

20. Bellver J, Ayllon Y, Ferrando M, Melo M, Goyri E, Pellicer A, et al. Female obesity impairs in vitro fertilization outcome without affecting embryo quality. Fertil Steril. 2010;93(2):447-54. doi: 10.1016/j.fertnstert.2008.12.032. [PubMed: 19171335].

21. Ramlau-Hansen CH, Thulstrup AM, Nohr EA, Bonde JP, Sorensen TI, Olsen J. Subfecundity in overweight and obese couples. Hum Reprod. 2007;22(6):1634-7. doi:10.1093/humrep/dem035. [PubMed: 17344224]. 
22. van der Steeg JW, Steures P, Eijkemans MJ, Habbema JD, Hompes PG, Burggraaff JM, et al. Obesity affects spontaneous pregnancy chances in subfertile, ovulatory women. Hum Reprod. 2008;23(2):324-8. doi: 10.1093/humrep/dem371. [PubMed:18077317].

23. Pinborg A, Gaarslev C, Hougaard CO, Nyboe Andersen A, Andersen $\mathrm{PK}$, Boivin J, et al. Influence of female bodyweight on IVF outcome: a longitudinal multicentre cohort study of 487 infertile couples. Reprod Biomed Online. 2011;23(4):490-9. doi: 10.1016/j.rbmo.2011.06.010. [PubMed: 21856228].

24. Luke B, Brown MB, Stern JE, Missmer SA, Fujimoto VY, Leach R, et al. Female obesity adversely affects assisted reproductive technology (ART) pregnancy and live birth rates. Hum Reprod. 2011;26(1):245-52. doi: 10.1093/humrep/deq306. [PubMed: 21071489].

25. Kasum M, Oreskovic S, Cehic E, Lila A, Ejubovic E, Soldo D. The role of female obesity on in vitro fertilization outcomes. Gynecol Endocrinol. 2018;34(3):184-8. doi: 10.1080/09513590.2017.1391209. [PubMed: 29037105].

26. Pasquali R, Pelusi C, Genghini S, Cacciari M, Gambineri A. Obesity and reproductive disorders in women. Hum Reprod Update. 2003;9(4):35972. doi: 10.1093/humupd/dmg024. [PubMed: 12926529].

27. Talmor A, Dunphy B. Female obesity and infertility. Best Pract Res Clin Obstet Gynaecol. 2015;29(4):498-506. doi: 10.1016/j.bpobgyn.2014.10.014. [PubMed: 25619586].

28. Gaskins AJ. Recent advances in understanding the relationship between long- and short-term weight change and fertility. F1000Res. 2018;7. doi: 10.12688/f1000research.15278.1. [PubMed: 30416711]. [PubMed Central: PMC6206616].

29. Bray GA. Obesity and reproduction. Hum Reprod. 1997;12 Suppl 1:2632. doi:10.1093/humrep/12.suppl_1.26. [PubMed: 9403319].

30. Santoro N, Lasley B, McConnell D, Allsworth J, Crawford S, Gold EB, et al. Body size and ethnicity are associated with menstrual cycle alterations in women in the early menopausal transition: The Study of Women's Health across the Nation (SWAN) Daily Hormone Study.J Clin Endocrinol Metab. 2004;89(6):2622-31. doi: 10.1210/jc.2003-031578. [PubMed: 15181033].

31. Purcell SH, Moley KH. The impact of obesity on egg quality. J Assist Reprod Genet. 2011;28(6):517-24. doi: 10.1007/s10815-011-9592-y. [PubMed: 21625966]. [PubMed Central: PMC3158259].

32. Farooqi IS, Jebb SA, Langmack G, Lawrence E, Cheetham $\mathrm{CH}$, Prentice AM, et al. Effects of recombinant leptin therapy in a child with congenital leptin deficiency. N Engl J Med. 1999;341(12):879-84. doi: 10.1056/NEJM199909163411204. [PubMed: 10486419].

33. Jungheim ES, Schoeller EL, Marquard KL, Louden ED, Schaffer JE, Moley KH. Diet-induced obesity model: abnormal oocytes and persistent growth abnormalities in the offspring. Endocrinology. 2010;151(8):4039-46. doi: 10.1210/en.2010-0098. [PubMed: 20573727]. [PubMed Central: PMC2940512].

34. Luzzo KM, Wang Q, Purcell SH, Chi M, Jimenez PT, Grindler N, et al. High fat diet induced developmental defects in the mouse: oocyte meiotic aneuploidy and fetal growth retardation/brain defects. PLoS One. 2012;7(11). e49217. doi: 10.1371/journal.pone.0049217. [PubMed: 23152876]. [PubMed Central: PMC3495769].

35. Duggal PS, Van Der Hoek KH, Milner CR, Ryan NK, Armstrong DT, Magoffin DA, et al. The in vivo and in vitro effects of exogenous leptin on ovulation in the rat. Endocrinology. 2000;141(6):1971-6. doi: 10.1210/endo.141.6.7509. [PubMed: 10830279].

36. Wittemer C, Ohl J, Bettahar-Lebugle K, Viville S, Nisand I. A quantitative and morphological analysis of oocytes collected during 438 IVF cycles. J Assist Reprod Genet. 2000;17(1):44-50. doi: 10.1023/a:1009402130903. [PubMed: 10754783]. [PubMed Central: PMC3455195].

37. Jones KP, Hatasaka HH, Peterson CM, Aoki V, Campbell B, Carrell DT. Body Mass Index (BMI) Is Inversely Related to Intrafollicular hCG Levels and Pregnancy Rates of IVF Patients. Fertil Steril. 2000;74(3). doi: 10.1016/s0015-0282(00)01231-0.

38. van Swieten EC, van der Leeuw-Harmsen L, Badings EA, van der Linden PJ. Obesity and Clomiphene Challenge Test as predictors of outcome of in vitro fertilization and intracytoplasmic sperm injection. Gynecol Obstet Invest. 2005;59(4):220-4. doi: 10.1159/000084347. [PubMed: 15753618].

39. Salha O, Dada T, Sharma V. Influence of body mass index and self-administration of hCG on the outcome of IVF cycles: a prospective cohort study. Hum Fertil (Camb). 2001;4(1):37-42. doi: 10.1080/1464727012000199241. [PubMed:11591255].

40. Matalliotakis I, Cakmak H, Sakkas D, Mahutte N, Koumantakis G, Arici A. Impact of body mass index on IVF and ICSI outcome: a retrospective study. Reprod Biomed Online. 2008;16(6):778-83. doi: 10.1016/s14726483(10)60142-3. [PubMed: 18549686].

41. Dokras A, Baredziak L, Blaine J, Syrop C, VanVoorhis BJ, Sparks A. Obstetric outcomes after in vitro fertilization in obese and morbidly obese women. Obstet Gynecol. 2006;108(1):61-9. doi: 10.1097/01.AOG.0000219768.08249.b6. [PubMed:16816057].

42. Esinler I, Bozdag G, Yarali H. Impact of isolated obesity on ICSI outcome. Reprod Biomed Online. 2008;17(4):583-7. doi: 10.1016/s14726483(10)60249-0. [PubMed: 18854116].

43. Bilenka B, Ben-Shlomo I, Cozacov C, Gold CH, Zohar S. Fertility, miscarriage and pregnancy after vertical banded gastroplasty operation for morbid obesity. Acta Obstet Gynecol Scand. 1995;74(1):42-4. doi: 10.3109/00016349509009942. [PubMed: 7856431].

44. Nichols JE, Crane MM, Higdon HL, Miller PB, Boone WR. Extremes of body mass index reduce in vitro fertilization pregnancy rates. Fertil Steril. 2003;79(3):645-7. doi: 10.1016/s0015-0282(02)04807-0. [PubMed: 12620460].

45. Wattanakumtornkul S, Damario MA, Stevens Hall SA, Thornhill AR, Tummon IS. Body mass index and uterine receptivity in the oocyte donation model. Fertil Steril. 2003;80(2):336-40. doi: 10.1016/s00150282(03)00595-8. [PubMed: 12909496].

46. Dechaud H, Anahory T, Reyftmann L, Loup V, Hamamah S, Hedon B. Obesity does not adversely affect results in patients who are undergoing in vitro fertilization and embryo transfer. Eur J Obstet Gynecol Reprod Biol. 2006;127(1):88-93. doi: 10.1016/j.ejogrb.2005.12.009. [PubMed: 16417960].

47. Best D, Bhattacharya S. Obesity and fertility. Horm Mol Biol Clin Investig. 2015;24(1):5-10. doi: 10.1515/hmbci-2015-0023. [PubMed: 26351959].

48. Robker RL. Evidence that obesity alters the quality of oocytes and embryos. Pathophysiology. 2008;15(2):115-21. doi: 10.1016/j.pathophys.2008.04.004. [PubMed: 18599275].

49. Bastounis EA, Karayiannakis AJ, Syrigos K, Zbar A, Makri GG, Alexiou D. Sex hormone changes in morbidly obese patients after vertical banded gastroplasty. Eur Surg Res. 1998;30(1):43-7. doi: 10.1159/000008556. [PubMed: 9493693].

50. Cano F, Garcia-Velasco JA, Millet A, Remohi J, Simon C, Pellicer A. Oocyte quality in polycystic ovaries revisited: identification of a particular subgroup of women. J Assist Reprod Genet. 1997;14(5):25461. doi: 10.1007/BF02765826. [PubMed: 9147238]. [PubMed Central: PMC3454720].

51. Gesink Law DC, Maclehose RF, Longnecker MP. Obesity and time to pregnancy. Hum Reprod. 2007;22(2):414-20. doi: 10.1093/humrep/del400. [PubMed: 17095518]. [PubMed Central: PMC1924918].

52. Wise LA, Rothman KJ, Mikkelsen EM, Sorensen HT, Riis A, Hatch EE. An internet-based prospective study of body size and timeto-pregnancy. Hum Reprod. 2010;25(1):253-64. doi: 10.1093/humrep/dep360. [PubMed: 19828554]. [PubMed Central: PMC2794667].

53. Chambers TJ, Richard RA. The impact of obesity on male fertility. Hormones (Athens). 2015;14(4):563-8. doi: 10.14310/horm.2002.1621. [PubMed: 26732149].

54. Campbell JM, Lane M, Owens JA, Bakos HW. Paternal obesity negatively affects male fertility and assisted reproduction out- 
comes: a systematic review and meta-analysis. Reprod Biomed Online. 2015;31(5):593-604. doi: 10.1016/j.rbmo.2015.07.012. [PubMed: 26380863].

55. Eisenberg ML, Kim S, Chen Z, Sundaram R, Schisterman EF, Buck Louis GM. The relationship between male BMI and waist circumference on semen quality: data from the LIFE study. Hum Reprod. 2014;29(2):193200. doi:10.1093/humrep/det428. [PubMed: 24306102]. [PubMed Central: PMC3896223].

56. Tsao CW, Liu CY, Chou YC, Cha TL, Chen SC, Hsu CY. Exploration of the association between obesity and semen quality in a 7630 male population. PLoS One. 2015;10(3). e0119458. doi: 10.1371/journal.pone.0119458. [PubMed: 25822490]. [PubMed Central: PMC4379020].

57. McPherson NO, Lane M. Male obesity and subfertility, is it really about increased adiposity? Asian J Androl. 2015;17(3):450-8. doi: 10.4103/1008-682X.148076. [PubMed: 25652636]. [PubMed Central: PMC4430951].

58. Mushtaq R, Pundir J, Achilli C, Naji O, Khalaf Y, El-Toukhy T. Effect of male body mass index on assisted reproduction treatment outcome: an updated systematic review and meta-analysis. Reprod Biomed Online. 2018;36(4):459-71. doi: 10.1016/j.rbmo.2018.01.002. [PubMed: 29452915].

59. Katib A. Mechanisms linking obesity to male infertility. Cent European J Urol. 2015;68(1):79-85. doi: 10.5173/ceju.2015.01.435. [PubMed: 25914843]. [PubMed Central: PMC4408383].

60. Liu Y, Ding Z. Obesity, a serious etiologic factor for male subfertility in modern society. Reproduction. 2017;154(4):R123-31. doi: 10.1530/REP17-0161. [PubMed: 28747541].

61. Guo D, Wu W, Tang Q, Qiao S, Chen Y, Chen M, et al. The impact of BMI on sperm parameters and the metabolite changes of seminal plasma concomitantly. Oncotarget.2017;8(30):48619-34. doi:10.18632/oncotarget.14950. [PubMed: 28159940]. [PubMed Central: PMC5564712].

62. Nguyen RH, Wilcox AJ, Skjaerven R, Baird DD. Men's body mass index and infertility. Hum Reprod.2007;22(9):2488-93. doi:10.1093/humrep/dem139. [PubMed: 17636282].

63. Sallmen M, Sandler DP, Hoppin JA, Blair A, Baird DD. Reduced fertility among overweight and obese men. Epidemiology. 2006;17(5):5203. doi: 10.1097/01.ede.0000229953.76862.e5. [PubMed:16837825].

64. Du Plessis SS, Cabler S, McAlister DA, Sabanegh E, Agarwal A. The effect of obesity on sperm disorders and male infertility. Nat Rev Urol. 2010;7(3):153-61. doi: 10.1038/nrurol.2010.6. [PubMed: 20157305].

65. Colaci DS, Afeiche M, Gaskins AJ, Wright DL, Toth TL, Tanrikut C, et al. Men's body mass index in relation to embryo quality and clinical outcomes in couples undergoing in vitro fertilization. Fertil Steril. 2012;98(5):1193-9 e1. doi: 10.1016/j.fertnstert.2012.07.1102. [PubMed 22884013]. [PubMed Central: PMC3478419].

66. Petersen GL, Schmidt L, Pinborg A, Kamper-Jorgensen M. The in fluence of female and male body mass index on live births after assisted reproductive technology treatment: a nationwide register-based cohort study. Fertil Steril. 2013;99(6):1654-62. doi: 10.1016/j.fertnstert.2013.01.092. [PubMed: 23394781].

67. Bellver J, Pellicer A, Garcia-Velasco JA, Ballesteros A, Remohi J, Meseguer M. Obesity reduces uterine receptivity: clinical experience from 9,587 first cycles of ovum donation with normal weight donors. Fertil Steril. 2013;100(4):1050-8. doi: 10.1016/j.fertnstert.2013.06.001. [PubMed: 23830106].

68. Jungheim ES, Schon SB, Schulte MB, DeUgarte DA, Fowler SA, Tuuli MG. IVF outcomes in obese donor oocyte recipients: a systematic review and meta-analysis. Hum Reprod. 2013;28(10):2720-7. doi: 10.1093/humrep/det292. [PubMed: 23847110]. [PubMed Central: PMC3777569].

69. DeUgarte DA, DeUgarte CM, Sahakian V. Surrogate obesity negatively impacts pregnancy rates in third-party reproduction. Fertil Steril. 2010;93(3):1008-10. doi: 10.1016/j.fertnstert.2009.07.1005. [PubMed: 19733847].

70. Styne-Gross A, Elkind-Hirsch K, Scott RJ. Obesity does not impact implantation rates or pregnancy outcome in women attempting conception through oocyte donation. Fertil Steril.2005;83(6):1629-34. doi: 10.1016/j.fertnstert.2005.01.099. [PubMed:15950629].

71. Anifandis G, Dafopoulos K, Messini CI, Polyzos N, Messinis IE. The BMI of men and not sperm parameters impact on embryo quality and the IVF outcome. Andrology. 2013;1(1):85-9. doi: 10.1111/j.20472927.2012.00012.x. [PubMed: 23258634].

72. Zhu J, Tang W, Mao J, Li J, Zhuang X, Liu P, et al. Effect of male body mass index on live-birth sex ratio of singletons after assisted reproduction technology. Fertil Steril. 2015;104(6):1406-10 e1-2. doi 10.1016/j.fertnstert.2015.08.017. [PubMed: 26361206].

73. Sheiner E, Menes TS, Silverberg D, Abramowicz JS, Levy I, Katz M, et al. Pregnancy outcome of patients with gestational diabetes mellitus following bariatric surgery. Am J Obstet Gynecol. 2006;194(2):431-5. doi:10.1016/j.ajog.2005.08.056. [PubMed:16458641].

74. Norman RJ, Mol BWJ. Successful weight loss interventions before in vitro fertilization: fat chance? Fertil Steril. 2018;110(4):581-6. doi 10.1016/j.fertnstert.2018.05.029. [PubMed: 30196941]. 